\title{
Catheter-over-needle and fixation method for interscalene block reduces risk of catheter dislocation; retrospective analysis from single center
}

\author{
Alper Tunga DOGAN*, Omur ERCELEN**, Deniz AKBAY***, \\ Feyzi ARTUKOGLU*** \\ Department of Anesthesiology, Amerikan Hospital Istanbul Turkey \\ KOC ÜNIVERSITESI \\ HASTANESI \\ *DESA-Anesthesiology Specialist, ${ }^{* *}$ Professor, ${ }^{* * *}$ Anesthesiology Specialist
}

AMERIKAN

AASTANESI

Interscalene nerve block is an effective method of pain management after shoulder surgery. Continuous nerve blocks provide long duration of analgesia and reduced use of opiods. However mechanical displacement of these catheters is still a problem. We would like to share our experience of interscalene catheters and offer a fixation method we currently use which reduced our catheter dislocation rates.

We analyzed 513 interscalene catheter insertions between January 2013- May 2018. Traditional catheter-through-needle (Contiplex-S, BBraun) technique was used until June 2015 at our institution. Catheter displacement and leakage were significant problems. We changed our practice to catheter-over-needle (Contiplex-C, BBraun) method and also start using catheter fixation set (Perifix Catheter fixation set, BBRaun). Catheter fixation set has a fixation ring and cover foil with non-adhesive center. At the end of the procedure film dressing (3M,Tegaderm) applied. All catheter placements done with ultrasound guidance and out-of-plane technique.
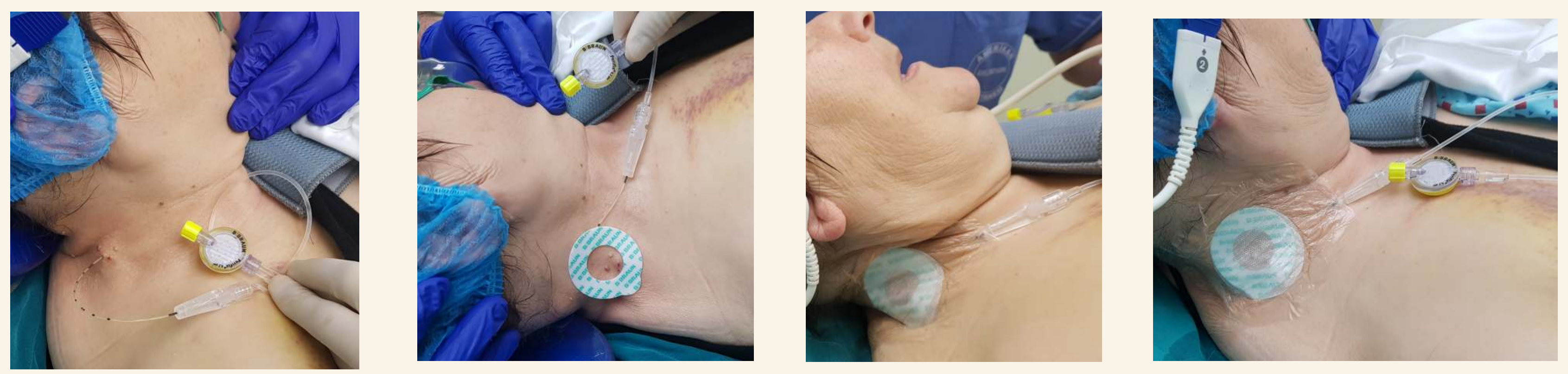

In this retrospective analysis we share our 6 years of experience. Incidence of mechanical displacement of interscalene catheters are reduced within years. Dislocation rates (\%)/years are as follows (Table). With the use of catheter-over-needle method none of the patients experienced leakage problem.

\begin{tabular}{|c|c|c|c|c|c|c|}
\hline & 2013 & 2014 & 2015 & 2016 & 2017 & 2018 \\
\hline Dislocation rates (\%) & 25 & 26 & 15 & 8 & 8 & 5 \\
\hline Patients number & 77 & 75 & 90 & 99 & 120 & 52 \\
\hline
\end{tabular}

In summary, use of catheter-over-needle method and fixation set reduced mechanical displacement of the interscalene catheter. This technique also reduces leakage rates and the risk of contamination. 